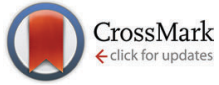

Cite this: J. Mater. Chem. C, 2016, 4, 3018

Received 28th October 2015, Accepted 22nd January 2016

DOI: $10.1039 /$ c5tc03541d

www.rsc.org/MaterialsC

\section{A fluorescent molecular rotor showing vapochromism, aggregation-induced emission, and environmental sensing in living cells $\dagger$}

\author{
Matthias Koenig, ${ }^{a}$ Barbara Storti, ${ }^{b}$ Ranieri Bizzarri, ${ }^{b}$ Dirk M. Guldi, ${ }^{a}$ \\ Giuseppe Brancato*c and Giovanni Bottari*de
}

\begin{abstract}
Among the plethora of recently proposed molecular sensors, those belonging to the class of fluorescent molecular rotors (FMRs) have attracted much attention owing to their peculiar photophysical properties that enable an unprecedented sensitivity towards environmental microviscosity. The usual FMR synthetic design prescribes chromophores characterized by an intramolecular rotation between two well-defined excited states, a locally excited state and a twisted internal charge transfer (TICT) state, where the sensing capabilities arise from a dual competition of the corresponding radiative/non-radiative decay processes. However, we have recently demonstrated a different modus operandi of a new subclass of solvatochromic FMRs, which exploit a solvent-independent, barrier-free intramolecular rotation of the excited dye. The rotational dynamics is modulated by local viscosity and, in turn, manifested through a variable spectral signal. In order to translate the same rotational mechanism in a versatile sensor of polarity and viscosity, we designed and thoroughly characterized a novel FMR, namely 4-(triphenylamino)-phthalonitrile (TPAP). Remarkably, in addition to a high sensitivity versus solvent polarity and viscosity, TPAP is also able to form stable fluorescent nanoparticles characterized by aggregation-induced emission, via a simple sonochemical treatment. Such peculiar features are tested in different applications aiming at illustrating its capability to report on solvatochromic and vapochromic effects, as well as to provide detailed intracellular information through bioimaging studies.
\end{abstract}

\section{Introduction}

Fluorophores whose emission changes in response to variations in their environment have emerged as an interesting family of compounds with widespread applications in analytical, environmental, optical, and biochemical areas. In this context, sensors for polarity and viscosity are the most expedient ones, owing to their widespread applicability in several life-science studies. ${ }^{1}$ The polarity sensitivity is generally based on the change in the dipole moment of the fluorophore upon photo-excitation and the resulting dipolar relaxation of the surrounding molecules or

\footnotetext{
${ }^{a}$ Department of Chemistry and Pharmacy \& Interdisciplinary Center for Molecular Materials (ICMM), Friedrich-Alexander-Universität Erlangen-Nürnberg, 91058, Erlangen, Germany

${ }^{b}$ NEST, Istituto Nanoscienze-CNR and Scuola Normale Superiore, Piazza San Silvestro 12, I-56127 Pisa, Italy

${ }^{c}$ Scuola Normale Superiore, Piazza dei Cavalieri 7, I-56126 Pisa, Italy. E-mail: giuseppe.brancato@sns.it

d Departamento de Química Orgánica, Universidad Autónoma de Madrid, 28049, Cantoblanco, Spain. E-mail: giovanni.bottari@uam.es

${ }^{e}$ IMDEA-Nanociencia, Campus de Cantoblanco, C/Faraday 9, 28049 Madrid, Spain $\dagger$ Electronic supplementary information (ESI) available: Additional photophysical studies, computational details. See DOI: 10.1039/c5tc03541d
}

residues. This effect is called solvatochromism and is accounted for by several theoretical descriptions, among which stands the Lippert-Mataga model ${ }^{2}$ for its simplicity and effective interpretation of many experimental data. ${ }^{3-5}$

Fluorophores sensitive to local viscosity are referred to as fluorescent molecular rotors (FMRs). ${ }^{6}$ FMRs perceive local viscosity through intramolecular twisting motion(s) that occur at the excited state. ${ }^{7}$ In most cases, intramolecular distortion leads to a twisted internal charge transfer (TICT) state, which is almost non-emissive. Thus, slowing the twisting motions as viscosity increases leads to stronger emission and longer lifetime decay. ${ }^{8}$ The ForsterHoffmann model of molecular rotors ${ }^{9}$ affords a reliable quantitative description of fluorescence dependence on viscosity for most FMRs. ${ }^{10}$ Nonetheless, a common drawback of FMRs is the difficulty to predict their sensitivity towards local polarity, owing to the complex dipolar properties of accessible excited states. ${ }^{11}$ Some FMRs are even insensitive to the dielectric properties of their surrounding environment. ${ }^{12}$ In this context, probes reporting predictably on both polarity and viscosity are a desirable tool in the field of fluorescence molecular sensors, since they may allow for thorough physicochemical characterization of their local environment. 
a)

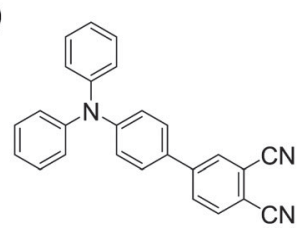

b)

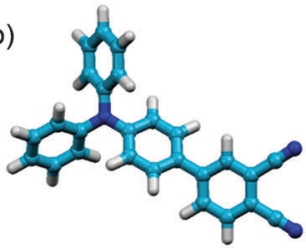

Fig. 1 (a) Molecular and (b) optimized structure of TPAP

We recently described a prototype for a new class of molecular rotors, namely 4-(diphenylamino)-phthalonitrile (DPAP), which combines strong fluorochromism and viscosity response within one molecule. ${ }^{13}$ This unique feature is attributed to an unprecedented and distinctive modus operandi, which is based on an unusual barrier-less and solvent-independent flexibility that finely modulates its photophysical properties. Motivated by these findings, we here present 4-(triphenylamino)-phthalonitrile (TPAP, Fig. 1a), a FMR designed to exploit the same atypical mechanism with an increased charge transfer distance. TPAP is easily synthesized in a few steps via a simple and convergent route and makes use of an amino electron donor unit and two nitrile electron acceptor units to facilitate intramolecular charge transfer (ICT) upon photoexcitation. The extended $\pi$-conjugated system of TPAP is designed to facilitate the formation of a charge transfer state, which, in turn, does affect its electronic and spectroscopic features, as well as its sensitivity towards the environment. In this work, we embarked on a thorough photophysical and computational study to analyze TPAP solvatochromism and viscosity response. On this account, TPAP has been tested in three different applications requiring environmental sensing. Our goal was to assess the feasibility and modus operandi of TPAP in different contexts, including its capability to report fluorescence emission and lifetime differences in the cellular environment.

First, TPAP was shown to exhibit excellent vapochromic response when exposed to a range of different solvent vapors. Hence, TPAP was demonstrated to give rise to the formation of highly-fluorescent organic nanoparticles based on an aggregation-induced emission (AIE) mechanism. ${ }^{14}$ Finally, TPAP was investigated as a dual polarity and viscosity probe in living cells, on account of the fundamental relevance of electrostatic interactions ${ }^{15-17}$ and diffusion-controlled reactions to sustain life. ${ }^{6}$ The sensing capability of TPAP is exploited by a new imaging approach, named phasor analysis, which relies on a simple graphic analysis of spectral and lifetime fluorescence images following a mathematical transformation. ${ }^{18}$ Overall, our results do support the use of TPAP as an outstanding probe in the field of environmental sensors.

\section{Materials and methods}

\section{Synthesis of TPAP}

TPAP was prepared and purified following the reported procedure. ${ }^{19}$ TPAP was characterized by ${ }^{1} \mathrm{H}$ and ${ }^{13} \mathrm{C}$ NMR, elemental analysis, mass spectrometry and IR, and the data obtained, that can be found in the ESI, $\dagger$ matched with the values reported in the literature.

\section{Preparation of TPAP AIE dispersions}

For the preparation of the TPAP dispersions in a 87/13 water/ methanol mixture, a solution of TPAP in methanol $(286 \mu \mathrm{L})$ was added to deionized water $(1914 \mu \mathrm{L})$ under vigorous stirring (final volume of the prepared solvents mixture $=2200 \mu \mathrm{L}$ ). A similar procedure, but using different ratios of the solution of TPAP in methanol and deionized water, has been used for the preparation of the other mixtures which present different water/ methanol ratios. The water/methanol mixtures at different TPAP concentrations (from $1.1 \times 10^{-6}$ up to $5.0 \times 10^{-4} \mathrm{M}$ ) were obtained using methanol solutions at different TPAP concentrations.

\section{Preparation of TPAP films for vapochromic studies}

TPAP films for vapochromic studies were prepared by uniformly spreading a hexane solution of TPAP on a glass slide and letting the solvent to evaporate. Residual traces of the solvent were eliminated by introducing the TPAP-coated glass slides in a vacuum chamber. The vapochromic studies were carried out by placing these coated slides into a chamber saturated with the vapours of different solvents while illuminating the slides with a $365 \mathrm{~nm}$ lamp.

\section{Cell cultures}

CHO cells were grown in Dulbecco's modified Eagle medium: F-12 nutrient mix (D-MEM/F-12) purchased from Invitrogen (Carlsbad, CA) supplemented with $10 \%$ fetal bovine serum and $100 \mathrm{U} \mathrm{ml}^{-1}$ penicillin, and $100 \mathrm{mg} \mathrm{ml}^{-1}$ streptomycin (Invitrogen). Cells were maintained at $37{ }^{\circ} \mathrm{C}$ in a humidified $5 \% \mathrm{CO}_{2}$ atmosphere. For fluorescence imaging, 120000 cells were plated onto a $35 \mathrm{~mm}$ glass-bottom dish (WillCo-dish GWSt-3522).

More details of quantum mechanical (QM) calculations, fluorescence microscopy imaging of living cells, photophysical studies of TPAP in solution and dispersions, cryogenic transmission electron microscopy (Cryo-TEM) studies and dynamic light scattering (DLS) measurements can be found in the ESI. $\dagger$

\section{Results and discussion}

\section{TPAP structural analysis}

The peculiar chemical structure of TPAP confers significant rotational freedom to its phenyl rings, while the backbone of the molecule is constrained to a Y-shaped configuration. From the computed optimized structure, it has been observed that the nitrogen atom is coplanar with its covalently linked carbon atoms, according to a $\mathrm{sp}^{2}$ hybridization. Due to steric hindrance, the amine phenyl groups assume a propeller-like configuration, as shown in Fig. $1 \mathrm{~b}$, and their rotational motion is therefore concerted. On the other hand, the 1,2-dicyanobenzene moiety can rotate rather freely with respect to the rest of the molecule (see Fig. S1, ESI $\dagger$ ), showing relatively small energetic barriers $\left(<3 \mathrm{kcal} \mathrm{mol}^{-1}\right)$ along the computed torsional potential.

\section{TPAP optical properties and solvatochromism}

Optical absorption and emission spectra of TPAP were recorded in various solvents and solvent mixtures of different polarity 


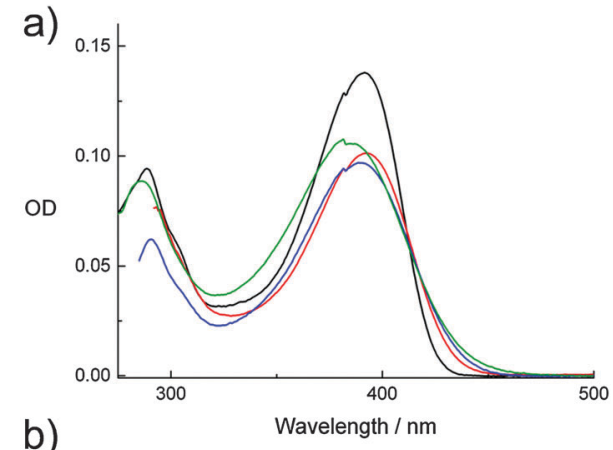

b)

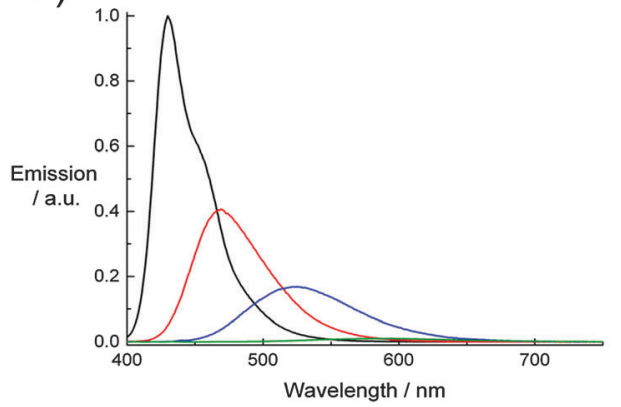

Fig. 2 (a) Absorption and (b) emission spectra $\left(\lambda_{\text {exc }}=392 \mathrm{~nm}\right)$ of TPAP ([TPAP] $=5 \times 10^{-6} \mathrm{M}$ and $1 \times 10^{-6} \mathrm{M}$ in (a) and (b), respectively) in cyclohexane (black), o-xylene (red), THF (blue) and acetonitrile (green).

(Table S1, ESI $\dagger$ ). The absorption spectra of TPAP in cyclohexane, $o$-xylene, THF and acetonitrile are shown in Fig. 2a. All spectra are characterized by two broad bands with maxima at about 390 and $285 \mathrm{~nm}$. QM calculations have assigned these bands to transitions from S0 to S1 and S4, respectively (see Fig. S2, ESI $\dagger$ for the computed optical spectra). Extinction coefficients of about $2 \times 10^{4} \mathrm{M}^{-1} \mathrm{~cm}^{-1}$ were observed in all solvents. However, a strong increase in absorption with extinction coefficients rising to $1 \times 10^{5} \mathrm{M}^{-1} \mathrm{~cm}^{-1}$ was noted in pyridine (Fig. S3, ESI $\dagger$ ). This finding may suggest that $\pi-\pi$ interactions with the solvent are responsible for the observed absorption enhancement.

Emission spectra recorded in cyclohexane, $o$-xylene, THF and acetonitrile are shown in Fig. 2b upon excitation at the absorption maximum (392 nm). In contrast to absorption, a strong solvatochromic effect is observed in the recorded emission spectra, affecting both emission energies and intensities. It is worth noting that a single, broad emission signal evolves in all solvents except for cyclohexane for which an additional small shoulder was detected. In this case, QM calculations have confirmed the nature of the emission as occurring from a relaxed S1 state and have nicely matched the observed solvatochromism in going from apolar to polar solvents (see Fig. S2, ESI $\dagger$ ).

For a detailed description of TPAP solvatochromism, the Lippert-Mataga polarity parameter $(\Delta f)$ was evaluated for an extended set of solvents and solvent mixtures according to eqn (S1) (Table S1, ESI $\dagger$ ). ${ }^{20,21}$ Fig. 3a shows the correlations between the optical absorption and emission maxima and $\Delta f$.

As previously shown for a few solvents, polarity has a little effect on the absorption maximum of TPAP, while the emission is associated with a significant solvatochromic effect. Remarkably, in strongly polar solvents, a Stokes shift as large as $200 \mathrm{~nm}$ is observed.
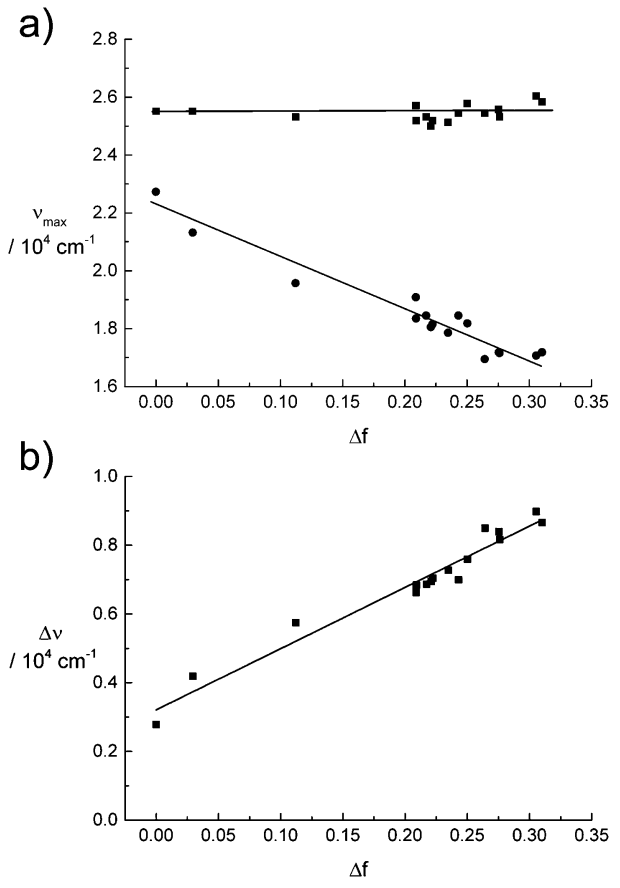

Fig. 3 (a) Plot of the absorption (squares) and emission (circles) maxima of TPAP as a function of the solvent polarity parameter $(\Delta f)$. (b) Lippert plot created by plotting the TPAP Stokes' shift $(\Delta v)$ as a function of $\Delta f$.

The good linear correlation with $\Delta f$ furthermore suggests that the nature of the fluorescent state is virtually equivalent in all applied solvents.

Next, to estimate the polarity sensitivity of TPAP, a Lippert plot was created by plotting the Stokes' shift against $\Delta f$ according to the Lippert-Mataga equation (eqn (1)), resulting in a linear correlation (Fig. $3 \mathrm{~b}$ ). Here, $\Delta \mu$ describes the change in the dipole moment upon excitation and can be used as a measure for solvent sensitivity of the fluorophore. ${ }^{21}$ From the slope of the Lippert plot, we could estimate a $\Delta \mu$ value of 17.4 D for TPAP (note that computed $\Delta \mu$ values for TPAP are $17.7 \mathrm{D}$ and $12.7 \mathrm{D}$ in acetonitrile and hexane, respectively). This significant dipole change is attributed to the strong ICT character of the excited state, owing to the extended $\pi$-system that enables a better charge separation between the oxidation and reduction centers of the molecule, and thereby increasing $\Delta \mu$ in a significant way.

$$
\Delta \nu=\Delta \nu_{0}+\frac{2\left(\mu_{\mathrm{e}}-\mu_{\mathrm{g}}\right)^{2}}{h c r^{3}} \Delta f
$$

Fluorescence quantum yields $(\Phi)$ and emission lifetimes $(\tau)$ of TPAP were determined in several solvents and are listed in Table S1 (ESI $\dagger$ ). $\Phi$ values of TPAP are generally very high in apolar solvents, while they are low $(<0.2)$ in polar and protic milieu (Fig. S4, ESI $\dagger$ ). $\Phi$ linearly decreases with increasing solvent polarity and, in turn, it confirms the trend established in the emission and Lippert plots.

All emission time decays were fitted by a mono-exponential function. Interestingly, $\tau$ values were found to increase linearly up to $\Delta f=0.22-0.25$, while in the high polarity region a sudden decrease set in (Fig. S5, ESI $\dagger$ ). This odd behaviour prompted us 


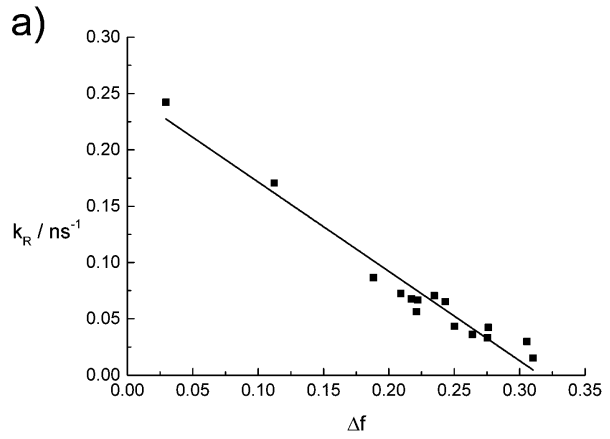

b)

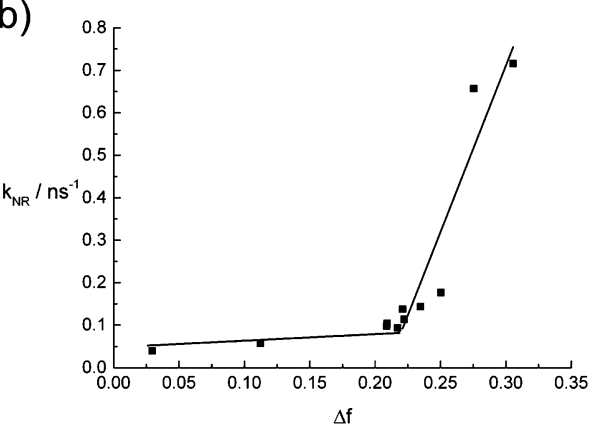

Fig. 4 Plot of the solvent parameter $\Delta f$ versus (a) the fluorescence radiative decay rate constant $\left(k_{R}\right)$ and (b) estimated non-radiative decay rate constant $\left(k_{\mathrm{NR}}\right)$ for TPAP.

to separate out the radiative $\left(k_{\mathrm{r}}\right)$ from the non-radiative $\left(k_{\mathrm{nr}}\right)$ decay rate constants by eqn (S2) (Table $\mathrm{S} 1$, ESI $\dagger$ ). $k_{\mathrm{r}}$ values showed good linearity when plotted versus $\Delta f$ (Fig. 4a). In contrast, $k_{\mathrm{nr}}$ vs. $\Delta f$ was associated with a different trend (Fig. 4b). For solvents with $\Delta f<0.22-0.25$, the polarity of the medium had no influence on $k_{\mathrm{nr}}$. However, in the high-polar solvent region $(\Delta f>0.22-0.25)$ a sudden increase in $k_{\mathrm{nr}}$ was detected. This effect was extremely pronounced in protic polar solvents such as methanol and ethylene glycol, suggesting a possible hydrogen-bonding stabilization of the ICT state.

Interestingly, besides the above mentioned solvatochromic response, TPAP also exhibits a strong vapochromic response. The emission wavelength of TPAP films $\left(\lambda_{\text {exc }}=365 \mathrm{~nm}\right)$ strongly varied when exposed to vapors of solvents with different polarity as observed by the naked eye (Fig. 5). In all cases, solvent vapors could be reversibly adsorbed by and removed from the films, with no apparent loss of emission efficiency. These results support the use of TPAP and structurally-related compounds $^{22}$ as new chromogenic materials suitable for the detection of volatile organic compounds (VOCs). Notably, the recent application of the hue parameter of the HSV color-space for spectral fluorescent sensing ${ }^{23}$ could open up the way to a cost-effective way to identify vapours and vapour mixtures by means of inexpensive RGB cameras.

\section{Characterization of the TPAP triplet state}

Nanosecond transient absorption experiments with excitation at $355 \mathrm{~nm}$ were carried out in oxygen-free THF solutions to characterize the triplet state of TPAP. The triplet state of TPAP showed a maximum at $700 \mathrm{~nm}$ that decayed over several $\mu$ s (Fig. S7, ESI $\dagger$ ).

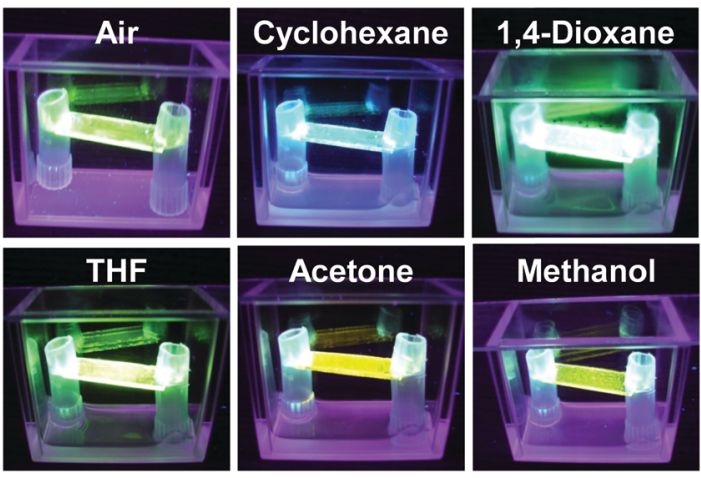

Fig. 5 Vapochromism of TPAP films on glass exposed to air and vapors of different solvents. Excitation wavelength for all samples was $365 \mathrm{~nm}$.

Quenching experiments with molecular oxygen confirmed the triplet nature of the detected absorption, with a quenching constant of $6.5 \times 10^{8} \mathrm{M}^{-1} \mathrm{~s}^{-1}$. Fitting the transient absorption decay traces resulted in a triplet lifetime of $26 \mu$ s for TPAP in THF (Fig. S7, inset, ESI $\dagger$ ).

In this context, singlet oxygen quantum yields, gathered at $1275 \mathrm{~nm}$ for TPAP in different solvents, helped us to confirm the solvent dependency of the triplet state formation (Fig. S8, ESI $\dagger$ ). ${ }^{24,25}$ The largest production of singlet oxygen was detected in $o$-xylene $\left(\Phi_{\Delta}=0.16\right)$, the less polar solvent, followed by THF $\left(\Phi_{\Delta}=0.13\right)$. Notably, comparison of these figures with $\Phi$ in the same apolar solvents indicated that intersystem crossing to triplet accounts for a large fraction of the non-radiative depopulation of the singlet excited state. Conversely, in polar solvents like acetonitrile almost no singlet oxygen could be detected $\left(\Phi_{\Delta}=0.01\right)$. The low $\Phi$ value of TPAP in polar solvents suggested that nonradiative decay channels connecting singlet excited and ground states become largely predominant.

\section{Transient absorption measurements on TPAP solutions}

To shed light on the excited state processes and the deactivation pathways of TPAP, femtosecond transient absorption spectroscopy was carried out in different solvents ( $o$-xylene, THF, acetonitrile and methanol). Photoexcitation (pump) was set at $387 \mathrm{~nm}$, and transient spectra were collected at different times in the 400-1100 $\mathrm{nm}$ range (Fig. 6). In all solvents, a large positive absorption appeared at $450-500 \mathrm{~nm}$; smaller positive bands were detected between $550-1100 \mathrm{~nm}$ (Fig. 6a). Notably, the band in the $500-600 \mathrm{~nm}$ region was found to change in $<10 \mathrm{ps}$, witnessing the dipolar relaxation of the surrounding solvent molecules (i.e., $1-10 \mathrm{ps}$ is the characteristic time of apolar solvent rearrangement around solvatochromic dyes). ${ }^{26}$

Owing to its intensity, the high-energy absorption at 450$500 \mathrm{~nm}$ was followed over time from 1 to $3500 \mathrm{ps}$. Time-plots of absorption at $480 \mathrm{~nm}$ showed faster kinetics in polar and protic solvents (acetonitrile and methanol) as compared to mediumpolarity or apolar milieu (Fig. 6b). In all cases, the transient profile decayed almost biexponentially (Table S2, ESI $\dagger$ ). The faster component (rate constant $k_{1}$ ) referred to a 10-100 ps kinetic process. The slower component (rate constant $k_{2}$ ) was 


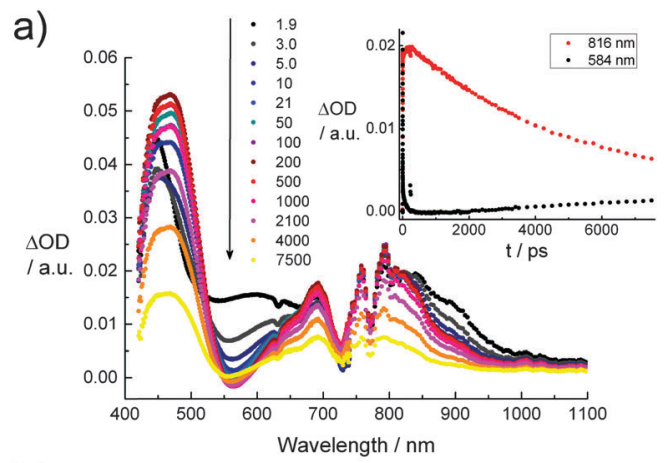

b)

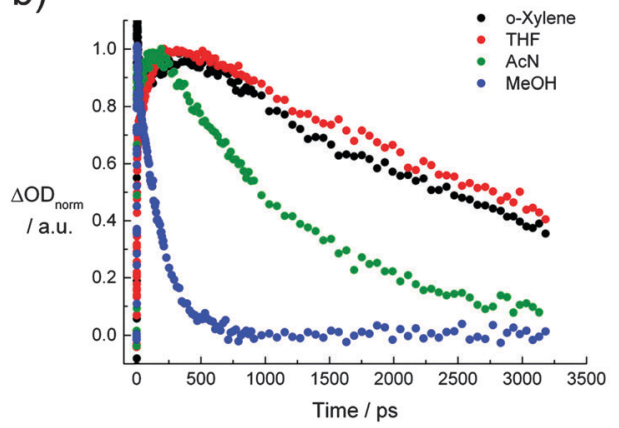

Fig. 6 (a) Differential absorption spectra of TPAP obtained upon femtosecond flash photolysis in argon-saturated THF with several time delays between 0 and 7500 ps (see legend for details). OD at the $387 \mathrm{~nm}$ excitation wavelength was $0.2-0.3$. Inset: Time-absorption profiles in THF monitoring intersystem crossing with singlet decay (red) and triplet formation (black). (b) Time-absorption profiles in o-xylene (black), THF (red), acetonitrile (green) and methanol (blue) monitored at $480 \mathrm{~nm}$.

attributed to the return to the ground state and was characterized by a time constant similar to fluorescence lifetime values. The plot of $k_{1}$ and $k_{2} v s$. the solvent polarity parameter $\Delta f$ resembled the relationship established for the non-radiative decay constants $k_{\mathrm{NR}}-$ vide supra (Fig. S6, ESI $\dagger$ ). These findings highlight the strong influence of solvent polarity in determining ICT formation and depopulation.

\section{Viscosity dependence of TPAP fluorescence}

Along with a strong solvatochromism, TPAP also presents a good response to the solvent viscosity $(\eta)$, especially in polar and protic solvents where the non-radiative deactivation of the excited state is predominant (vide supra). Increasing the solvent viscosity should lead to a decrease in the rotor's flexibility with a smaller interval and lower total values of the average dihedral angle being accessible. This influences the rotor's deactivation pathways by shifting towards a more radiative decay. ${ }^{27}$

Experimentally, we measured fluorescence emission, in media characterized by high and constant polarity values and variable viscosities. Fig. 7 shows the emission changes for TPAP in protic solvents such as methanol $(\eta=0.52$ at $298 \mathrm{~K})$, ethylene glycol ( $\eta=16.2$ at $298 \mathrm{~K})$, and glycerine $(\eta=950$ at $298 \mathrm{~K})$. Upon increasing the solvent viscosity, we observed a clear increase of emission intensity accompanied by a significant blue-shift. It is worth noting that the shift of the emission wavelength upon changing viscosity is generally not observed for TICT rotors,

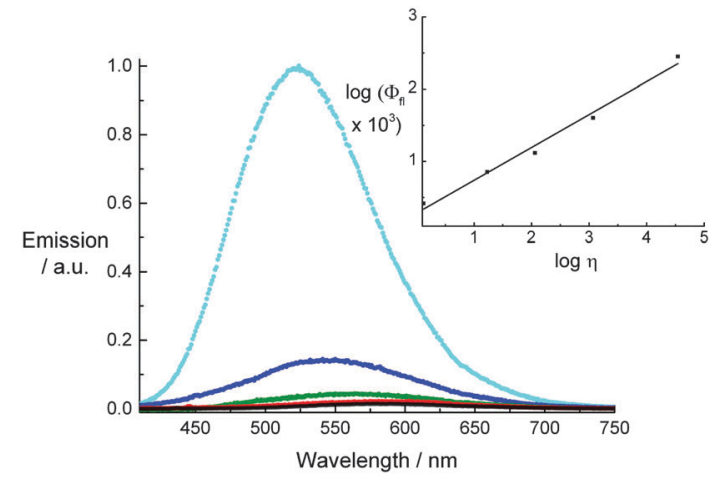

Fig. 7 Emission of TPAP in solvents of different viscosity: methanol (black), ethylene glycol (red), ethylene glycol/glycerine (1:1) (green), glycerine at $295 \mathrm{~K}$ (blue), and glycerine at $265 \mathrm{~K}$ (cyan) The optical density at the $395 \mathrm{~nm}$ excitation wavelength was 0.09 for all samples. Inset: The double-logarithmic plot of the solvent viscosity $(\eta)$ versus the fluorescent quantum yield $\left(\Phi_{\mathrm{fl}}\right)$ for TPAP according to the Förster-Hoffmann equation.

where emission emerges from an energetically invariable locallyexcited state. $^{10}$

Next, we investigated further the system plotting $\eta v s$. $\Phi_{\mathrm{fl}}$ for five solvents/solvent mixtures. Data complied with the FörsterHoffmann equation (eqn (2)) (Fig. 7, inset), ${ }^{28}$ which predicts linearity on a double logarithmic scale on account of the response of conformational motions to the solvent viscosity. TPAP shows high sensitivity towards viscosity changes as witnessed by the Förster-Hoffmann slope $x=0.46$. Notably, this value is in agreement with the figures determined for most fluorescent rotors. ${ }^{29}$

$$
\log \Phi_{\mathrm{fl}}=x \log \eta+C
$$

As high solvent polarity mainly affects the non-radiative decay pattern, transient absorption spectroscopy experiments were additionally carried out by inspecting the absorption band located at $480 \mathrm{~nm}$. Upon increasing the viscosity from methanol to glycerol, lifetimes increased significantly by a factor of 10 for the short-lived compound $k_{1}$ (formation) and by a factor of 4 for the longer-lived compound $k_{2}$ (decay) (Table S2, ESI $\dagger$ ). Similarly, a double-logarithmic plot according to the modified FörsterHoffmann equation was created and revealed a linear dependency in the investigated viscosity interval for both $k_{1}$ and $k_{2}$ (Fig. S9, ESI $\dagger$ ).

\section{Aggregation-induced emission studies on TPAP}

To evaluate the dependence of TPAP emission upon aggregation, we investigated TPAP nanoparticles formed in aqueous media using the reprecipitation method. ${ }^{30}$

Notably, the fluorescence of TPAP was found to quench upon addition of a small amount of water to a methanol solution. This effect was attributed to the increase in solvent polarity that favoured non-radiative deactivation. However, when the water content exceeded $50 \%(\mathrm{v} / \mathrm{v})$, the emission gradually intensified reaching a maximum for a water content of around $70 \%$ (Fig. 8 , inset). The astounding emission increase (120-fold) was attributed to TPAP aggregation and it largely surpasses the emission 


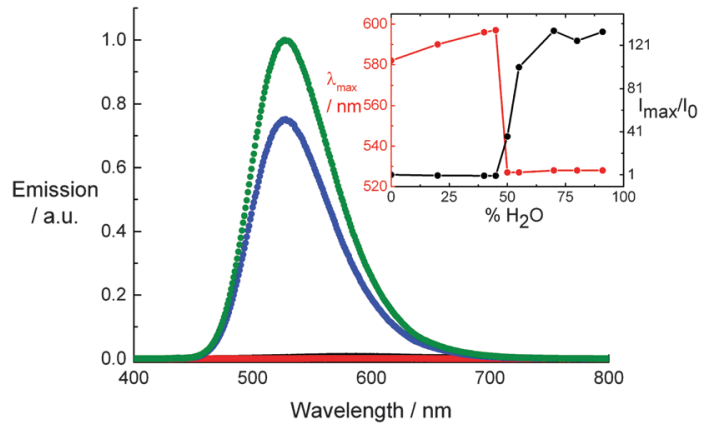

Fig. 8 Fluorescence emission $\left(\lambda_{\text {exc }}=390 \mathrm{~nm}\right)$ of TPAP ([TPAP] $=4.5 \times$ $10^{-5} \mathrm{M}$ ) in methanol (black) and in 45/55 (v/v) (red), 55/45 (v/v) (blue) and $87 / 13$ (v/v) (green) water/methanol mixtures. Inset: Relative emission intensity (right) and emission maximum wavelength (left) of water/methanol mixtures of TPAP as a function of the water content.

enhancement obtained with DPAP (a 20-fold emission increase). ${ }^{31}$ At the same time, a blue-shift of the emission maximum of $c a$. $70 \mathrm{~nm}$ (from 600 to $528 \mathrm{~nm}$ ) and a red shift of the absorption maximum from 390 (methanol) to $414 \mathrm{~nm}(87 / 13(\mathrm{v} / \mathrm{v})$ water/ methanol mixture) were noticed. Absorption spectra were accompanied by a broad tail reaching almost $800 \mathrm{~nm}$, which gives further evidence to the formation of TPAP aggregates (Fig. S10, ESI $\dagger$ ). In addition, upon freezing the TPAP water/methanol mixture, a further emission increase ( $c a$. 1.3-fold) was observed (Fig. 9). ${ }^{32,33}$

Lifetime studies helped to better rationalize the observed photophysical behaviour. As compared to methanol solution ( $\tau=0.17 \mathrm{~ns}$ ), fluorescence lifetime increased up to $12.9 \mathrm{~ns}$ for the AIE dispersions and even further (14.3 ns) upon freezing the TPAP dispersion (Fig. S13 and Table S3, ESI $\dagger$ ). The lifetime increase was consistent with the increase in their emission intensities (vide supra). The emission for TPAP nanoparticle dispersions decays biexponentially. Time-resolved emission spectroscopy (TRES) highlighted a clear blue-shift of about $20 \mathrm{~nm}$ in the $0.5-20 \mathrm{~ns}$ time range. This finding suggested the presence of a faster component emitting at shorter wavelengths and a slower component emitting at longer wavelengths (Fig. S14, ESI $\dagger$ ).

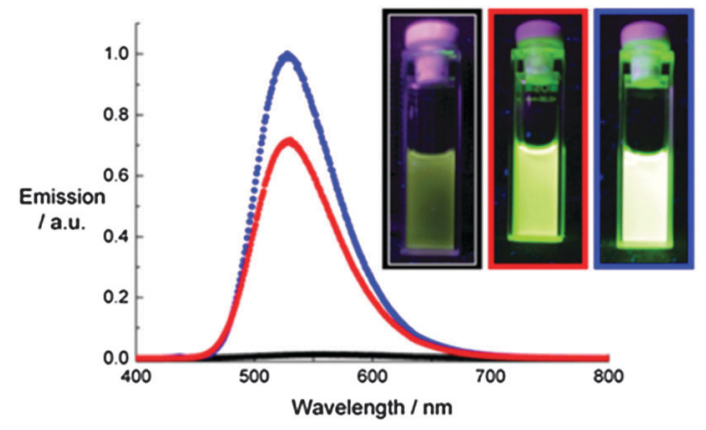

Fig. 9 Fluorescence emission $\left(\lambda_{\text {exc }}=390 \mathrm{~nm}\right)$ of TPAP $\left(4.5 \times 10^{-5} \mathrm{M}\right)$ in methanol (black) and $87 / 13(\mathrm{v} / \mathrm{v})$ water/methanol mixtures at room temperature (red) and frozen (green). TPAP concentration in both solution and dispersions is $4.5 \times 10^{-5} \mathrm{M}$. Inset: Pictures of the three cuvettes used for the fluorescent experiments upon illumination at $365 \mathrm{~nm}$. The coloured boxes around each picture follow the same colour-code used for the fluorescence experiments.
Indeed, an analysis of the deconvoluted, time-resolved emission spectra corroborated this concept (Fig. S15, ESI $\dagger$ ). From these observations and literature data, ${ }^{34,35}$ we speculated that in the TPAP aggregates the local charge-transfer within a monomer is accompanied by the intermolecular delocalization of the chargeseparated state which deactivates via a long-lived and red-shifted emission.

Dispersions at different TPAP concentrations were prepared in an $87 / 13(\mathrm{v} / \mathrm{v})$ water/methanol mixture. ${ }^{36}$ These studies showed that AIE set in above $1 \mu \mathrm{M}$ TPAP (Fig. S11, ESI $\dagger$ ), which is much lower compared to the figure relevant to DPAP $(20 \mu \mathrm{M})$. TPAP dispersions were found to be reasonably stable for a few hours; also, ultrasonication did not prompt changes in structure, emission and stability.

The structural properties of TPAP aggregates were studied using dynamic light scattering (DLS) and cryogenic-transmission electron microscopy (cryo-TEM) techniques. DLS showed that the TPAP aggregates correspond to nanoparticles with radius below $300 \mathrm{~nm}$. The nanoparticle size decreased upon increasing the water content of the dispersing medium (Fig. 10). On the other hand, TPAP concentration did not affect the nanoparticle radius (Fig. S12, ESI $\dagger$ ).

Consistent with DLS results, cryo-TEM on $87 / 13$ (v/v) water/ methanol highlighted both spherical and fragmented materials with an average size of 100-200 nm (Fig. 11).

\section{TPAP as an environmental fluorescent probe in living cells}

The last set of experiments established TPAP as an environmental probe in living cells, on account of its sensitivity to local viscosity and polarity. At first, TPAP was added to the external medium of Hamster Ovary Cells (CHOs) and its intracellular distribution was evaluated by confocal imaging. Within a few minutes from administration, TPAP was found to internalize effectively inside the cells, without affecting their viability. The dye mostly distributed in the endoplasmic reticulum (ER), and in internalization vesicles (Fig. 12). This partition into internal membrane organelles follows the moderate lipophilic nature of TPAP, and it is in agreement with the intracellular distribution of other neutral and lipophilic dyes. ${ }^{37}$

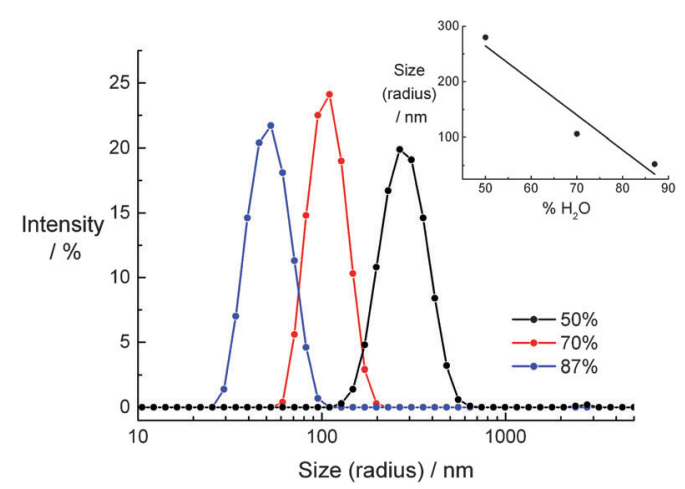

Fig. 10 Dynamic light scattering (DLS) measurements of TPAP dispersions ([TPAP] $\left.=4.5 \times 10^{-5} \mathrm{M}\right)$ in different water/methanol mixtures $(50 / 50$ $(\mathrm{v} / \mathrm{v})($ black), $70 / 30(\mathrm{v} / \mathrm{v})(\mathrm{red})$ and $87 / 13(\mathrm{v} / \mathrm{v})(\mathrm{blue}))$. Inset: Plot of the average hydrodynamic radius vs. the water content. 


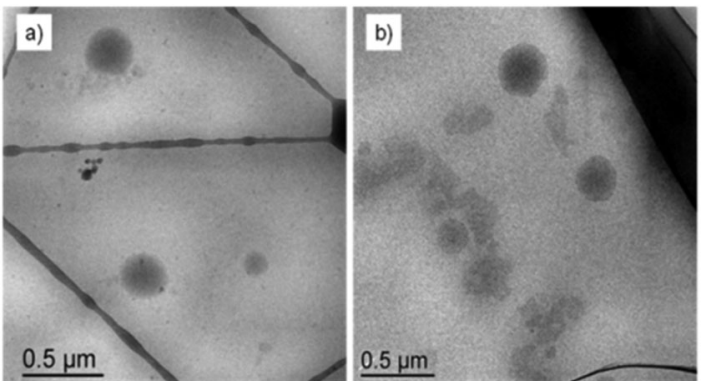

Fig. 11 (a and b) Cryo-TEM pictures of 87/13 (v/v) water/methanol dispersions of TPAP at $4.5 \times 10^{-5} \mathrm{M}$.

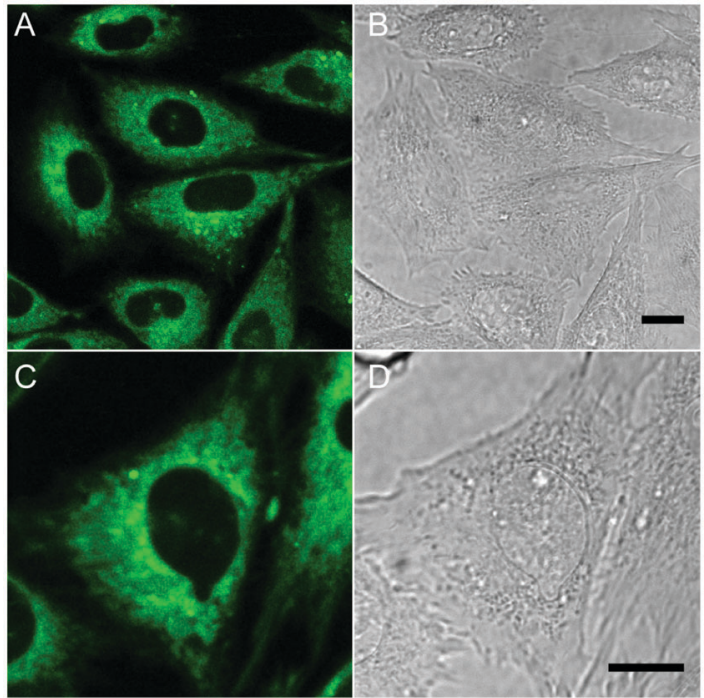

Fig. 12 (A and C) Fluorescence confocal images revealing intracellular localization of TPAP in CHO cells and (B and D) corresponding transmission images. Scale bar in B and D: $10 \mu \mathrm{m}$.

The ability of TPAP to evaluate local polarity and viscosity was assessed by two complementary techniques, which independently monitor the spectral and lifetime features of the probe, respectively. Both techniques are based on the "phasor analysis" approach developed by Gratton's group. ${ }^{42,43}$ In phasor analysis, the spectral or the lifetime profile of each pixel is Fourier-transformed to produce a vector (phasor) represented in a $2 \mathrm{D}$ plot named the "phasor plot". Two cartesian (G and S) or polar (phase and modulation) coordinates pinpoint any phasor. In the spectral phasor plot, the angular coordinate (phase) is proportional to the spectral center of mass; the radial coordinate (modulation) is inversely related to the spectral width. A red-shift in wavelength leads to an increase of the angular position, whereas a decrease in the spectral width increases the radius of the phasor. In the lifetime phasor plot, longer lifetimes are associated with larger phase and smaller modulation values. Notably, monoexponential lifetimes map out onto a semicircle of radius $1 / 2$ and center $(1 / 2,0)$.

A cluster of points in any phasor plot corresponds to pixels with similar spectra or lifetimes. Phasor clusters in different positions identify different population/structures in target samples.
Thus, the phasor approach provides an ideal tool for hyper-spectral and/or lifetime imaging analysis. Importantly, phasor combinations follow simple vectorial addition rules. For instance, the phasor of a two-component mixture inevitably falls on a line connecting the two phasors of the pure species. The distance from the resultant phasor to the individual "reference" phasors is inversely proportional to the contribution of that component, allowing for the calculation of the intensity fraction associated with each species. Several groups, including us, applied successfully the phasor analysis to follow a broad range of intracellular processes. ${ }^{38,39}$

Intracellular polarity analysis by TPAP was carried out by sequentially collecting 57 fluorescence confocal images from 430 to $600 \mathrm{~nm}$ on a target cell region. Fig. 13A shows the sum of all images and represents the whole emission in the 430-600 nm range. Fig. 13B displays the spectral phasor plot relevant to the spectral acquisition. We investigated the phasor clouds relevant to two different cell organelles, namely internalization vesicles (Fig. 13A, cyan arrow) and the ER (Fig. 13A, yellow arrow). The pixels relevant to the two organelles (Fig. 13C) mapped out different phasor regions, which were enclosed by coloured squares in Fig. 13B. Notably, these regions were unlike in term of phase angles (ER: $-135^{\circ}$ to $-180^{\circ}$; vesicles: $-225^{\circ}$ to $-300^{\circ}$ ), indicating large spectral differences between these two organelles. Indeed, vesicles were associated with a significant wavelength blue-shift $\left(\lambda_{\max }=480 \mathrm{~nm}\right)$ as compared to ER $\left(\lambda_{\max }=500 \mathrm{~nm}\right)$ (Fig. 13D), although the Lippert-Mataga calibration of TPAP highlighted rather hydrophobic environments in both cases. These findings may suggest that TPAP sensed a lower polarity in vesicles
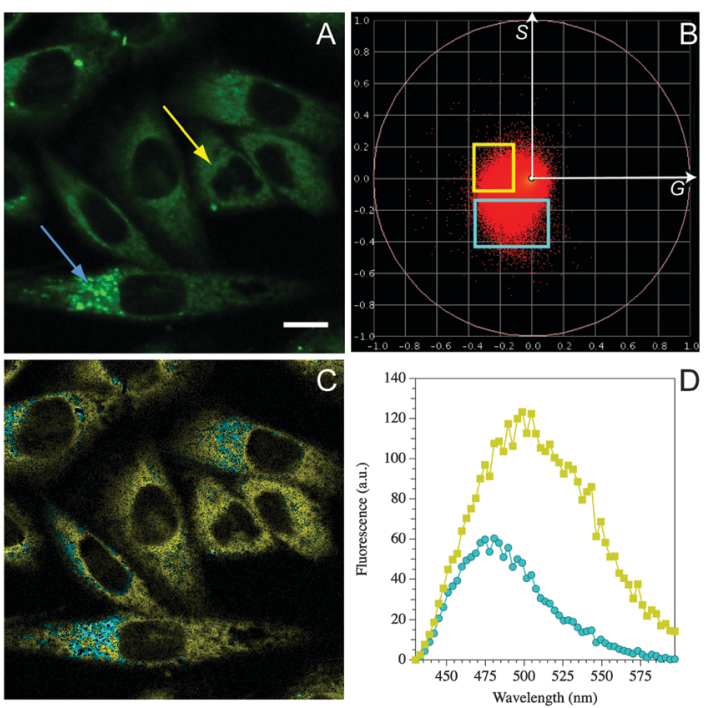

Fig. 13 Polarity analysis of living $\mathrm{CHO}$ cells by TPAP emission according to the phasor approach. (A) Global fluorescence confocal image of $\mathrm{CHO}$ cells obtained scanning the emission interval from 430 to $600 \mathrm{~nm}$ in 59 steps; two representative cell vesicle and ER regions are indicated by a cyan and a yellow arrow, respectively; (B) spectral phasor of the $430-600 \mathrm{~nm}$ stack of images: two distinguishable phasor regions were enclosed in two colored squares; (C) phasor separation in the confocal image: the pixels corresponding to phasors in the cyan and yellow squares of panel (B) are colored accordingly, highlighting cell vesicles and the ER; (D) spectra of phasor regions enclosed in the cyan and yellow squares: each spectrum has the same color code. Scale bar in (A): $10 \mu \mathrm{m}$. 
than in the ER. Alternatively, the higher TPAP concentration in vesicles (see intensity differences in Fig. 13A) led to significant dye aggregation and yielded a blue-shift similar to that observed for TPAP nanoparticles (vide supra).

Next, we carried out intracellular viscosity measurements by inspecting the fluorescence lifetime of TPAP. Fig. 14A reports the collective lifetime phasor plot relevant to 19 cell fields. Notably, TPAP showed phasor clouds falling within the universal circle (Fig. 14A). This indicates that TPAP emission is no more monoexponential. Nonetheless, multiexponential decays are often encountered when rotors are embedded in complex cellular domains and reflect the environmental heterogeneity at the nanoscale. ${ }^{40,41}$ A second striking feature of the phasor clouds was their almost linear distribution. This arrangement prompted us to consider each TPAP phasor as an intensity-weighted sum of the two reference phasors lying on the universal circle, on account of the vector additivity rule of phasors. ${ }^{42}$ The reference phasors $\mathrm{H}$ and $\mathrm{L}$ were identified by the intersections between the universal circle and the linear fit of the global phasor cloud associated with all normal cells.

As a matter of fact, this approach corresponds to a graphical deconvolution of the lifetime decay into two monoexponential components $\left(\tau_{\mathrm{H}}=4.37 \mathrm{~ns}, \tau_{\mathrm{L}}=1.47 \mathrm{~ns}\right)$ for each pixel of any image. ${ }^{43}$

$\mathrm{H}$ and $\mathrm{L}$ identify two limit conditions of high and low local viscosities, respectively. ${ }^{41}$ The map of intensity fraction relevant to $\mathrm{H}\left(X_{\mathrm{H}}\right)$ was calculated for each image by simple vector algebra. ${ }^{44}$ The inspection of the $\mathrm{H}$ maps (Fig. 14D and G) provided a simple
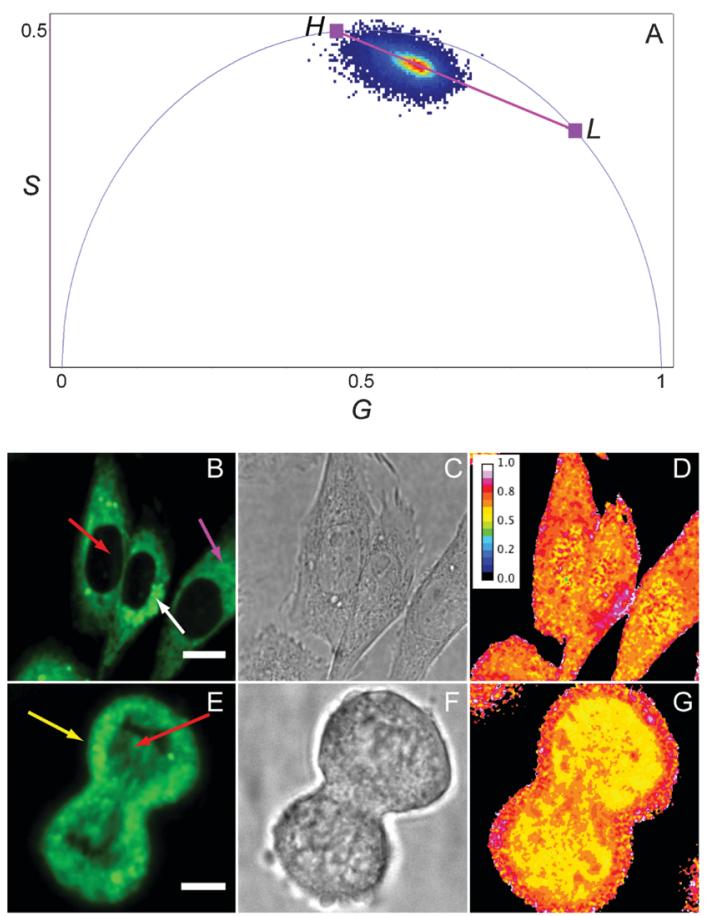

Fig. 14 Viscosity analysis of living $\mathrm{CHO}$ cells by TPAP lifetime according to the phasor approach. (A) Collective lifetime phasor plot relevant to 19 cell images; the calibration line connecting the two reference phasors $H$ and $L$ is also reported; ( $B$ and $E$ ) fluorescence confocal images and corresponding ( $C$ and $F$ ) transmission images and ( $D$ and $G$ ) maps of $H$ intensity fraction $\left(X_{H}\right)$. Scale bars: (B) $10 \mu \mathrm{m}$, (E) $5 \mu \mathrm{m}$. and straightforward way to compare relative viscosities of intracellular domains. TPAP entrapped in internalization vesicles (Fig. 14B, white arrow) sensed higher viscosity $\left(\left\langle X_{\mathrm{H}}\right\rangle=0.827 \pm\right.$ 0.043) than in the ER (Fig. 14B, purple arrow; $\left\langle X_{\mathrm{H}}\right\rangle=0.707 \pm$ 0.036). Consistently, endosome vesicle membranes are about 3 times richer in cholesterol (the most relevant stiffening factor of biomembranes) than the ER. ${ }^{45}$ Higher viscosity could also be brought about by dye aggregation in vesicles, inducing concomitantly a blue-shift of emission as discussed before.

Even more remarkable was the capability of TPAP to report the local viscosity of regions where its fluorescence is rather dim, namely chromatin in the cell nucleus (Fig. 14B and E, red arrow), and plasma membranes (Fig. 14E, yellow arrow). In this context, the dividing cell of Fig. 14E-G nicely shows the fluidity of the chromatin environment $\left(\left\langle X_{\mathrm{H}}\right\rangle=0.598 \pm 0.038\right)$ as compared with the rigid environment of plasma membranes $\left(\left\langle X_{\mathrm{H}}\right\rangle=0.732 \pm 0.068\right)$.

\section{Conclusions}

In summary, in this work we described the complete photophysical and computational characterization of TPAP, a new FMR. TPAP represents a derivative of highly-flexible molecular rotors, which exert barrier-less rotations independent of the solvent. Therefore, it belongs to the same FMR family of DPAP, as its modus operandi lacks the presence of a TICT state. Instead, the emissive deactivation of the solvent-stabilized ICT state is either accompanied by triplet formation in non- and mediumpolar solvents, or by direct non-radiative conversion into the ground state in polar and protic solvents. As compared to its parent analog (DPAP), TPAP is 2-3 fold more emissive and it is associated with a solvatochromism that spans an interval of $150 \mathrm{~nm}$, resulting in Stokes' shifts larger than $200 \mathrm{~nm}$ in high polar solvents. At the same time, a sensitive viscosity dependence of the TPAP emission was established. Hence, with the help of spectroscopic and microscopic techniques, information on the emissive and structural properties of TPAP nanoparticles has also been gathered. In particular, TPAP nanoparticles show a notable enhancement of emission intensity with lifetimes of up to $14 \mathrm{~ns}$, as obtained from time-resolved studies, which is more than twice as long as the lifetime of TPAP molecules in solution. On account of TPAP's peculiar ability to report on local polarity and viscosity, we applied the probe to image confocally these features at the intracellular level using a simple and straightforward graphic method named phasor analysis. Pleasantly, TPAP returned relative polarity and viscosity values of intracellular organelles (e.g., endosomes, endoplasmic reticulum) that are in line with their compositions and structures. Further information on biological systems might be accessible by targeting specific subcellular regions via biofunctionalization of the rotor.

\section{Acknowledgements}

This work was supported by MIUR through FIRB (contract no. RBFR10DAK6 and RBFR12ETL5) and PRIN (contract no. 
2012SK7ASN and 2010BJ23MN_004) projects. DREAMS Lab technical staff is kindly acknowledged for managing the computing facilities at SNS.

\section{Notes and references}

1 (a) C. Reichardt, Chem. Rev., 1994, 94, 2319-2358; (b) G. Signore, R. Nifosi, L. Albertazzi, B. Storti and R. Bizzarri, J. Am. Chem. Soc., 2010, 132, 1276-1288; (c) G. S. Loving, M. Sainlos and B. Imperiali, Trends Biotechnol., 2010, 2, 73-83; (d) X. Peng, Z. Yang, J. Wang, J. Fan, Y. He, F. Song, B. Wang, S. Sun, J. Qu, J. Qi and M. Yan, J. Am. Chem. Soc., 2011, 133, 6626-6635; (e) A. Battisti, S. Panettieri, G. Abbandonato, E. Jacchetti, F. Cardarelli, G. Signore, F. Beltram and R. Bizzarri, Anal. Bioanal. Chem., 2013, 405, 6223-6233; $(f)$ G. Brancato, G. Signore, P. Neyroz, D. Polli, G. Cerullo, G. Abbandonato, L. Nucara, V. Barone, F. Beltram and R. Bizzarri, J. Phys. Chem. B, 2015, 119, 6144-6154.

2 N. Mataga, Y. Kaifu and M. Koizumi, Bull. Chem. Soc. Jpn., 1956, 29, 465-470.

3 D. Marsh, Biophys. J., 2009, 96, 2549-2558.

4 A. K. Satpati, M. Kumbhakar, S. Nath and H. Pal, Photochem. Photobiol., 2009, 85, 119-129.

5 H. Weitman, M. Roslaniec, A. A. Frimer, M. Afri, D. Freeman, Y. Mazur and B. Ehrenberg, Photochem. Photobiol., 2001, 73, 110-118.

6 M. K. Kuimova, Phys. Chem. Chem. Phys., 2012, 14, 12671-12686.

7 M. A. Haidekker and E. A. Theodorakis, Org. Biomol. Chem., 2007, 5, 1669-1678.

8 M. T. Stockl, R. Bizzarri and V. Subramaniam, in Properties and Functions of Biological Membranes Investigated by Fluorescence Methods, ed. Y. Mély and G. Duportail, Springer, Berlin Heidelberg, 2012, vol. 3, pp. 215-240.

9 T. Forster and G. Hoffmann, Z. Phys. Chem., 1971, 75, 63-76.

10 M. A. Haidekker and E. A. Theodorakis, J. Biol. Eng., 2010, 4, 11.

11 M. A. Haidekker, T. P. Brady, D. Lichlyter and E. A. Theodorakis, Bioorg. Chem., 2005, 33, 415-425.

12 M. A. H. Alamiry, A. C. Benniston, G. Copley, K. J. Elliott, A. Harriman, B. Stewart and Y. G. Zhi, Chem. Mater., 2008, 20, 4024-4032.

13 M. Koenig, G. Bottari, G. Brancato, V. Barone, D. M. Guldi and T. Torres, Chem. Sci., 2013, 4, 2502-2511.

14 (a) J. Luo, Z. Xie, J. W. Y. Lam, L. Cheng, H. Chen, C. Qiu, H. S. Kwok, X. Zhan, Y. Liu, D. Zhu and B. Z. Tang, Chem. Commun., 2001, 1740-1741; (b) Y. Hong, J. W. Y. Lam and B. Z. Tang, Chem. Soc. Rev., 2011, 40, 5361-5388; (c) Y. Hong, J. W. Y. Lam and B. Z. Tang, Chem. Commun., 2009, 4332-4353; (d) J. Mei, N. L. C. Leung, R. T. K. Kwok, J. W. Y. Lam and B. Z. Tang, Chem. Rev., 2015, 115, 11718-11940.

15 A. Warshel and A. Dryga, Proteins, 2011, 79, 3469-3484.

16 A. Warshel, P. K. Sharma, M. Kato and W. W. Parson, Biochim. Biophys. Acta, 2006, 1764, 1647-1676.

17 D. A. Cherepanov, B. A. Feniouk, W. Junge and A. Y. Mulkidjanian, Biophys. J., 2003, 85, 1307-1316.
18 M. Stefl, N. G. James, J. A. Ross and D. M. Jameson, Anal. Biochem., 2011, 410, 62-69.

19 L. Giribabu, V. K. Singh, Ch. Vijay Kumar, Y. Soujanya, P. Yella Reddy and M. Lakshmi Kantam, Sol. Energy, 2011, 85, 1204-1212.

$20 \Delta f$ is reasonably suitable to describe the effects in non-protic solvents, where specific solvent solute interactions such as hydrogen bonding, etc. are absent. However, these specific solvent effects can be identified as deviations from the theory.

21 J. R. Lakowicz, Principles of Fluorescence Spectroscopy, Plenum Press, New York, 2006.

22 P. Minei, M. Koenig, A. Battisti, M. Ahmad, V. Barone, T. Torres, D. M. Guldi, G. Brancato, G. Bottari and A. Pucci, J. Mater. Chem. C, 2014, 2, 9224-9232.

23 A. Hakonen, J. E. Beves and N. Stromberg, Analyst, 2014, 139, 3524-3527.

24 Singlet oxygen quantum yields are used as a measure for triplet excited state generation.

25 A. A. Abdel-Shafi and F. Wilkinson, Phys. Chem. Chem. Phys., 2002, 4, 248-254.

26 L. Reynolds, J. A. Gardecki, S. J. V. Frankland, M. L. Horng and M. Maroncelli, J. Phys. Chem., 1996, 100, 10337-10354.

27 (a) K. Y. Law, Chem. Phys. Lett., 1980, 75, 545-549; (b) R. O. Loutfy and B. A. Arnold, J. Phys. Chem., 1982, 86, 4205-4211.

28 T. Foerster and G. Z. Hoffmann, Z. Phys. Chem., 1971, 75, 63-76.

29 J. Sutharsan, D. Lichlyter, N. E. Wright, M. Dakanali, M. A. Haidekker and E. A. Theodorakis, Tetrahedron, 2010, 66, 2582-2588.

30 H. Nakanishi and H. Oikawa, Reprecipitation method for organic nanocrystals, in Single Organic Nanoparticles, ed. H. Masuhara, H. Nakanishi and K. Sasaki, Springer-Verlag, Berlin, 2003, pp. 17-31.

31 M. Koenig, T. Torres, V. Barone, G. Brancato, D. M. Guldi and G. Bottari, Chem. Commun., 2014, 50, 12955-12958.

32 Considering the ICT nature of TPAP emission, we ascribe this effect to the decrease of non-radiative charge recombination processes upon lowering the temperature.

33 (a) C. M. Heller, I. H. Campbell, B. K. Laurich, D. L. Smith, D. D. C. Bradley, P. L. Burn, J. P. Ferraris and K. Mullen, Phys. Rev. B: Condens. Matter Mater. Phys., 1996, 54, 5516-5522; (b) Y. V. Ryabchikov, S. A. Alekseev, V. V. Lysenko, G. Bremond and J. M. Bluet, Nanoscale Res. Lett., 2012, 7, 365-369.

34 Such a dual emission has been previously observed in molecular aggregates of aromatic fluorophores and it has been attributed to emission from monomeric, localized and delocalized excited states.

35 (a) K. Inoue and M. Itoh, Bull. Chem. Soc. Jpn., 1979, 1, 45-48; (b) P. P. Neelakandan and D. Ramaiah, Angew. Chem., Int. Ed., 2008, 47, 8407-8411.

36 Unless otherwise stated, $87 / 13(\mathrm{v} / \mathrm{v})$ is the optimal solvent ratio used for the preparation of the water/methanol TPAP dispersions.

37 G. Signore, G. Abbandonato, B. Storti, M. Stockl, V. Subramaniam and R. Bizzarri, Chem. Commun., 2013, 49, 1723-1725.

38 E. Hinde, M. A. Digman, K. M. Hahn and E. Gratton, Proc. Natl. Acad. Sci. U. S. A., 2013, 110, 135-140. 
39 A. Battisti, M. A. Digman, E. Gratton, B. Storti, F. Beltram and R. Bizzarri, Chem. Commun., 2012, 48, 5127-5129.

40 Y. L. Wu, M. Stefl, A. Olzynska, M. Hof, G. Yahioglu, P. Yip, D. R. Casey, O. Ces, J. Humpolickova and M. K. Kuimova, Phys. Chem. Chem. Phys., 2013, 15, 14986-14993.

41 G. Ferri, L. Nucara, T. Biver, A. Battisti, G. Signore and R. Bizzarri, Biophys. Chem., 2016, 208, 17-25.
42 D. M. Jameson, E. Gratton and R. D. Hall, Appl. Spectrosc. Rev., 1984, 20, 55-106.

43 M. A. Digman, V. R. Caiolfa, M. Zamai and E. Gratton, Biophys. J., 2008, 94, L14-16.

44 K. M. Hirshfield, D. Toptygin, B. S. Packard and L. Brand, Anal. Biochem., 1993, 209, 209-218.

45 G. van Meer, D. R. Voelker and G. W. Feigenson, Nat. Rev. Mol. Cell Biol., 2008, 9, 112-124. 